\title{
Anabases
}

ANABASES Traditions et réceptions de l'Antiquité

$26 \mid 2017$

Varia

\section{I documenti settecenteschi come strumenti per lo studio degli scavi ercolanesi}

Agnes Allroggen-Bedel

\section{OpenEdition}

1 Journals

Édition électronique

URL : http://journals.openedition.org/anabases/6285

DOI : 10.4000/anabases.6285

ISSN : 2256-9421

Éditeur

E.R.A.S.M.E.

Édition imprimée

Date de publication : 1 novembre 2017

Pagination : 103-116

ISSN : 1774-4296

\section{Référence électronique}

Agnes Allroggen-Bedel, « / documenti settecenteschi come strumenti per lo studio degli scavi ercolanesi », Anabases [En ligne], 26 | 2017, mis en ligne le 01 novembre 2020, consulté le 20 janvier 2021. URL : http://journals.openedition.org/anabases/6285 ; DOI : https://doi.org/10.4000/anabases.6285

(c) Anabases 


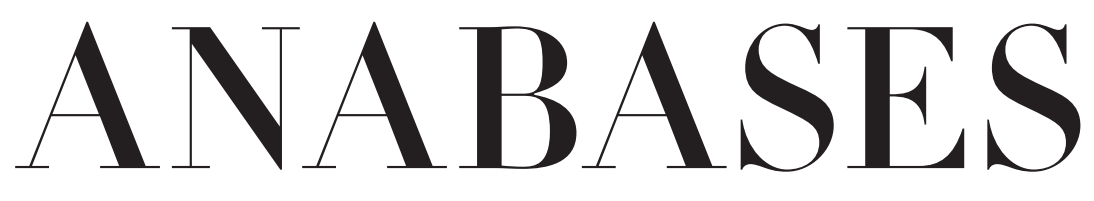

Traditions et Réceptions de l'Antiquité

$$
\begin{aligned}
& N \circ 26 \\
& 2017
\end{aligned}
$$

\section{E.R.A.S.M.E.}

Université Toulouse - Jean Jaurès 



\section{Sommaire}

$\mathrm{N}^{\circ} 26-2017$

\section{Historiographie et identités culturelles}

Jocelyne Peigney

La polysémie poétique d'anabase chez Saint-John Perse :

évocations, étymologie et botanique $\ldots \ldots \ldots \ldots \ldots \ldots \ldots \ldots \ldots \ldots$

Nicolas FAELLI

Les références antiques des fondateurs de l'Amérique française

au XVII siècle ................................. I9

Silvia Alaura

Setting the stage for Hittite studies in Victorian England:

practices and methods of the 1870 . . . . . . . . . . . . .

\section{Traditions du patrimoine antique}

“ Restituer Herculanum I. Des archives de fouilles aux restitutions 3D »

Alexandra Dardenay et Adeline Grand-Clément

Restituer Herculanum au XXI ${ }^{\mathrm{e}}$ siècle. L'apport des archives. . . . . . . . 55

Antonella Coralini

In situ e alibi, dallo scavo integrato alla cultura dell'abitare :

Vesuviana-DHER a Ercolano . . . . . . . . . . . . . . . . 67 67

Agnes Allroggen-Bedel

I documenti settecenteschi come strumenti per lo studio

degli scavi ercolanesi . . . . . . . . . . . . . . . Io3

James ANDREws

Revisiting the upper floors of the casa di Argo at Herculaneum . . . . . . II7 
Nicolas MonteIx

Les archives des fouilles d'Herculanum au XX $\mathrm{XX}^{\mathrm{e}}$ siècle :

analyse critique et pistes d'exploitation . . . . . . . . . . I43

\section{Archéologie des savoirs}

Yannick Le PAPE

L'inspiration et l'imprudence. Poésie de l'anticomanie

dans la critique d'art du second XIX ${ }^{\mathrm{e}}$ siècle . . . . . . . . . . . . . I57

\section{Actualités et débats}

Claude AzızA

Antiquités parallèles (7). La starlette des bandelettes . . . . . . . . . I77

Alexandra TrachseL

La réception d'Homère dans le domaine de la rhétorique $\ldots \ldots \ldots$. . . I83

Claude Aziza

Curiosa (2) In vino (vin/eau) veritas (very tasse) : drame de l'ivresse . . . . I89

\section{Lire, relire la bibliothèque des sciences de l'Antiquité}

Laurent OLIVIER

Pour en finir avec la race : Henri Hubert et l'anthroposociologie

À propos du commentaire de “L'Aryen, son rôle social », de Vacher de Lapouge $(\mathrm{r} 899) \ldots \ldots \ldots$

Henri Hubert

Compte rendu de Georges VACher de LAPouge, L'Aryen, son rôle social, L'Année sociologique, igoo, p. i45-146 . . . . . . . . . . . . . . . . . 203

\section{L'atelier de l'histoire : chantiers historiographiques}

L'Atelier des doctorants (coordonné par Adeline Grand-Clément) (14)

Francesco LoDÀ,

L'initiation des guerriers chez les peuples indo-européens.

Une enquête historiographique dans les pas de Georges Dumézil . . . 207

\section{Comptes rendus}

Benjamin Anderson et Robert G. Ousterhout

Palmyra I885. The Wolfe Expedition and the photographs

of John Henry Haynes (M. Sartre) . . . . . . . . . . . . . . . . . . . 2 215 
Mary R. BAchvarova

From Hittite to Homer. The Anatolian Background of Ancient Greek Epic (G. Hoffmann) . . . . . . . . . . . . . . . . .

Patrick Baker, Ronny Kaiser, Maike Priesterjahn, Johannes Helmrath (éd.) Portraying the Prince in the Renaissance. The Humanist Depiction of Rulers in Historiographical and Biographical Texts (G. Demelemestre). . 220

Brigitte BAKHоUсHE (éd.)

Science et exégèse. Les interprétations antiques et médiévales du récit biblique de la création des éléments (Genèse I, I-8) (F. P. BARONE) . . . . . . .

Laurence Baurain-Rebillard (éd.)

Héros grecs à travers le temps : autour de Persée, Thésée, Cadmos et Bellérophon (C. Giovénal) . . . . . . . . . . . . . . . .

Mark W. PAdiLla,

Classical myth in four films of Alfred Hitchcock . . . . . . . . . . . . . . .

Vivien Bessieres

Le Péplum et après? L’Antiquité gréco-romaine dans les récits contemporains (Cl. Aziza) . . . . . . . . . . . . . . . . . 225

Pierre Briant

Alexandre. Exégèse des lieux communs (É. Guillon) . . . . . . . . . . . . . 227

Paulo ButTi DE Lima

Il piacere delle Immagini. Un tema aristotelico nella riflessione moderna sull'arte (C. Noacco) . . . . . . . . . . . . . . . . . . .

Bénédicte Coudì̀re

La truelle et le phylactère. La proximité des images (M. Scapin) . . . . . . . 23I

Thorsten Fögen, Richard WARREN (éd.)

Graeco-Roman Antiquity and the Idea of Nationalism in the rgth Century, Case Studies (M. Champier) . . . . . . . . . . . . . . . ${ }_{232}$

Ronald H. Fritze

Egyptomania. A History of Fascination, Obsession and Fantasy

(Fabien Bièvre-Perrin) . . . . . . . . . . . . . . . . . . . . . 234

Joseph Geiger

Hellenism in the East. Studies on Greek Intellectuals in Palestine

(C. Bonnet). . . . . . . . . . . . . . . . . 236

Tiffany Jenkins

Keeping their marbles. How the treasures of the past ended up in museums... and why they should stay there (C. Valenti) . . . . . . . . . . 238 
Ayelet H. Lushkov

Magistracy and the Historiography of the Roman Republic.

Politics in Prose (C. Landrea) . . . . . . . . . . . . . . . . . . . . . . 240

Jean-Claude MüHLEthaler

Énée le mal-aimé. Du roman médiéval à la bande dessinée

(Fl. Bouchet) ........................ 24I

Laurent PERNoT

Epideictic Rhetoric, Questioning the Stakes of Ancient Praise

(C.Psilakis) ......................... 243

Priscian

Answers to King Khosroes of Persia (O. Gengler) . . . . . . . . . . . . . . 245

Paul Robertson

Paul's letters and contemporary Greco-Roman literature:

theorizing a new taxonomy (M. Cambron-Goulet) . . . . . . . . . . . . . . 247

Federico Santangelo (éd.)

Approaching the Roman Revolution. Sir Ronald Syme,

Papers on Republican History (C. Landrea) . . . . . . . . . . . . . . . . . . 249

Molly Swetnam-BurLand

Egypt in Italy. Visions of Egypt in Roman Imperial Culture

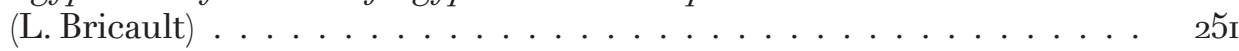

Helen VAN Noorden

Playing Hesiod. The 'Myth of the Races' in Classical Antiquity

(D. Bonanno)

Résumés . . . . . . . . . . . . . . . . . . . . . . . . . . . . . . . 257

Index .......................... . . 265 


\section{Traditions}

\section{du patrimoine antique}

Restituer Herculanum I.

Des archives de fouilles aux restitutions 3D 

Anabases 26 (2017), p. 103-116.

\section{I documenti settecenteschi come strumenti per lo studio degli scavi ercolanesi}

Agnes Allroggen-Bedel

documenti d'archivio relativi agli scavi settecenteschi nell'area vesuviana sono strumenti importanti per lo studio degli scavi, per la ricostituzione dei complessi pittorici e per la ricontestualizzazione degli oggetti provenienti dalle città vesuviane.

Gran parte di questa documentazione è stata pubblicata già nell'Ottocento. Negli ultimi decenni sono apparsi altri studi che consentono di analizzare i documenti settecenteschi senza dover consultare i manoscritti originali custoditi nei vari archivi napoletani e spagnoli.

Vanno ricordati soprattutto il libro di Michele Ruggiero, uscito nel 1885, "Scavi di Ercolano", ' e "Il Giornale degli scavi di Ercolano" pubblicato da Ulrico Pannuti nel 1983 con i rapporti di Don Rocco d'Alcubierre, primo scavatore di Ercolano, che vanno dal 1738 al $1756 .{ }^{2}$ Si tratta di una trascrizione del manoscritto intitolato "Noticia de las alajas que se han descubierto en las escavaciones de Resina y en otras ...", custodito nella biblioteca della Società Napoletana di Storia Patria, con un testo introduttivo, ma senza analisi archeologica. È da menzionare inoltre una raccolta di documenti riguardanti i primi anni degli scavi, curata da Mario Pagano. ${ }^{3}$

1 M. Ruggiero, Storia degli scavi di Ercolano ricomposta su'documenti superstiti, Napoli 1885.

2 U. Pannuti, “ Il diario di scavo di Ercolano 1738-1756 », Memorie dell'Accademia dei Lincei 3, 1983, pp. 163-410.

3 M. Pagano, I primi anni degli scavi di Ercolano, Pompei e Stabiae. Raccolta e studio di documenti e disegni inediti, Studi della Soprintendenza archeologica di Pompei 11, Roma, 2005. 
Nel 2006 Mario Pagano e Raffaele Prisciandaro hanno pubblicato due grandi volumi dal titolo "Studio sulle provenienze degli oggetti rinvenuti negli scavi borbonici del regno di Napoli", nei quali tentano di elencare tutti i rinvenimenti degli scavi borbonici del Sette- e de l'Ottocento sulla base dei diari di scavo. ${ }^{4}$

Si tratta di elenchi con la data di rinvenimento e un breve accenno al testo dei documenti, insieme ai numeri d'inventario degli oggetti individuati nel museo e ai luoghi di scavo. Infatti gli autori sono riusciti a individuare soprattutto molte pitture menzionate nei documenti settecenteschi. Manca però il riferimento alle ricerche già edite sull'argomento e alla situazione in situ. Spesso è difficile seguire il ragionamento degli autori, per capire come siano arrivati alle loro conclusioni, che vengono presentate come fatti assodati. La pubblicazione, che potrebbe essere uno strumento molto utile per la conoscenza dei primi decenni degli scavi di Ercolano, in taluni casi aumenta la confusione perché presuppone una affidabilità che purtroppo non c'è. È da augurarsi che dopo questo primo approccio segua una revisione critica.

Grazie a tutte queste iniziative, molti documenti settecenteschi sono oggi "a portata di mano", sebbene talvolta la loro lettura deluda le aspettative, soprattutto per quanto riguarda i primi decenni degli scavi ad Ercolano. Esaminando i rapporti di scavo, si incorre nel problema di capire in quale parte della città e in quale edificio si stava lavorando. Individuare gli oggetti menzionati nei rapporti di scavo, descritti spesso in modo abbastanza approssimativo, non è facile e talvolta addirittura impossibile. Mancano quasi tutti i disegni eseguiti sul posto e solo raramente ci sono indicazioni sulla situazione dei ritrovamenti in situ.

Nonostante queste difficoltà, i documenti settecenteschi offrono informazioni preziose che possono aiutare a recuperare, almeno virtualmente, decorazioni e contesti perduti. Per illustrare i problemi, il metodo, le possibilità e i limiti di tali studi, saranno presentati alcuni esempi significativi.

Prima di tutto, dobbiamo sottolineare che i primi decenni dopo la riscoperta di Ercolano nel 1738 furono un periodo di sperimentazione. Scavare un’intera città antica seppellita da un compatto banco di depositi vulcanici, fu un'impresa difficile e del tutto nuova. Si lavorava in cunicoli, come in una miniera, al buio, cercando di farsi strada attraverso il duro materiale vulcanico. Non a caso la documentazione degli scavi eseguiti a Pompei oppure a Stabia (iniziati nel 1748), dove si poteva scavare all'aperto, è molto più precisa e accurata.

4 M. Pagano, R. Prisciandaro, Studio sulle provenienze degli oggetti rinvenuti negli scavi borbonici del regno di Napoli: una lettura integrata, coordinata e commentata della documentazione, Studi e ricerche della Soprintendenza per i beni archeologici del Molise 1-2, Roma 2006. 


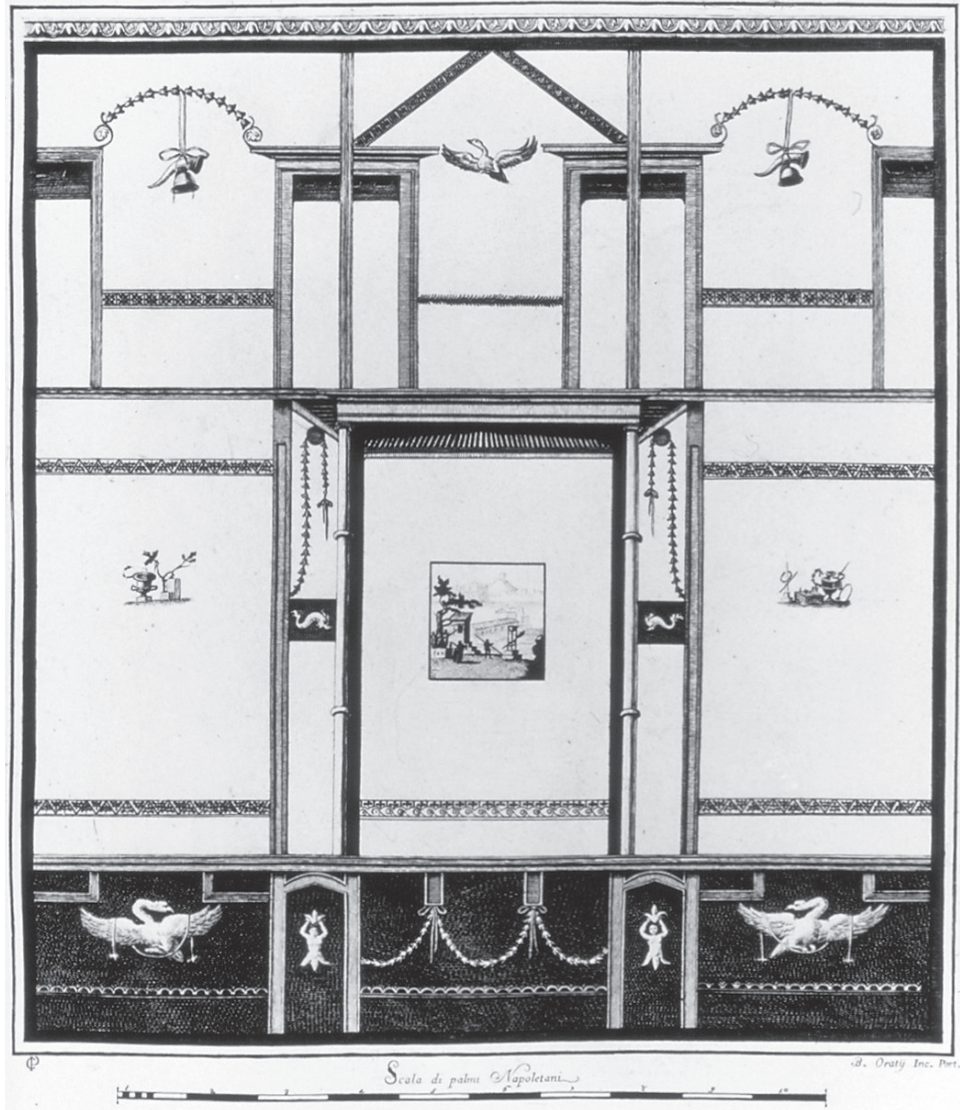

Fig. 1. Castellammare di Stabia, Villa San Marco, stanza 52, parete Est Da: Gli ornati delle pareti ed i pavimenti delle stanze dell'antica Pompei, incisi in rame, vol. II., Napoli 1808, tav. 25

Purtroppo della documentazione grafica di piante e disegni è rimasto ben poco. Sappiamo che alcune pareti furono disegnate, per documentare il loro contesto decorativo originario, prima dello stacco di singoli pezzi di pittura. Unico esempio di una tale documentazione sono i disegni di due pareti di un cubicolo nella Villa San Marco a Castellammare di Stabia che mostrano la decorazione ancora intatta. ${ }^{\mathbf{5}}$ (figg.1-2)

5 A. Allroggen-Bedel, “ Gli scavi borbonici nelle ville Stabiane: pitture antiche e gusto settecentesco ", in G. Bonifacio, A.M. Sodo (a cura di), Stabiae: Storia e Architettura. 250 Anniversario degli Scavi di Stabia, 1749-1999. Studi della Soprintendenza archeologica di Pompei 7, Roma 2002, p. 102. 


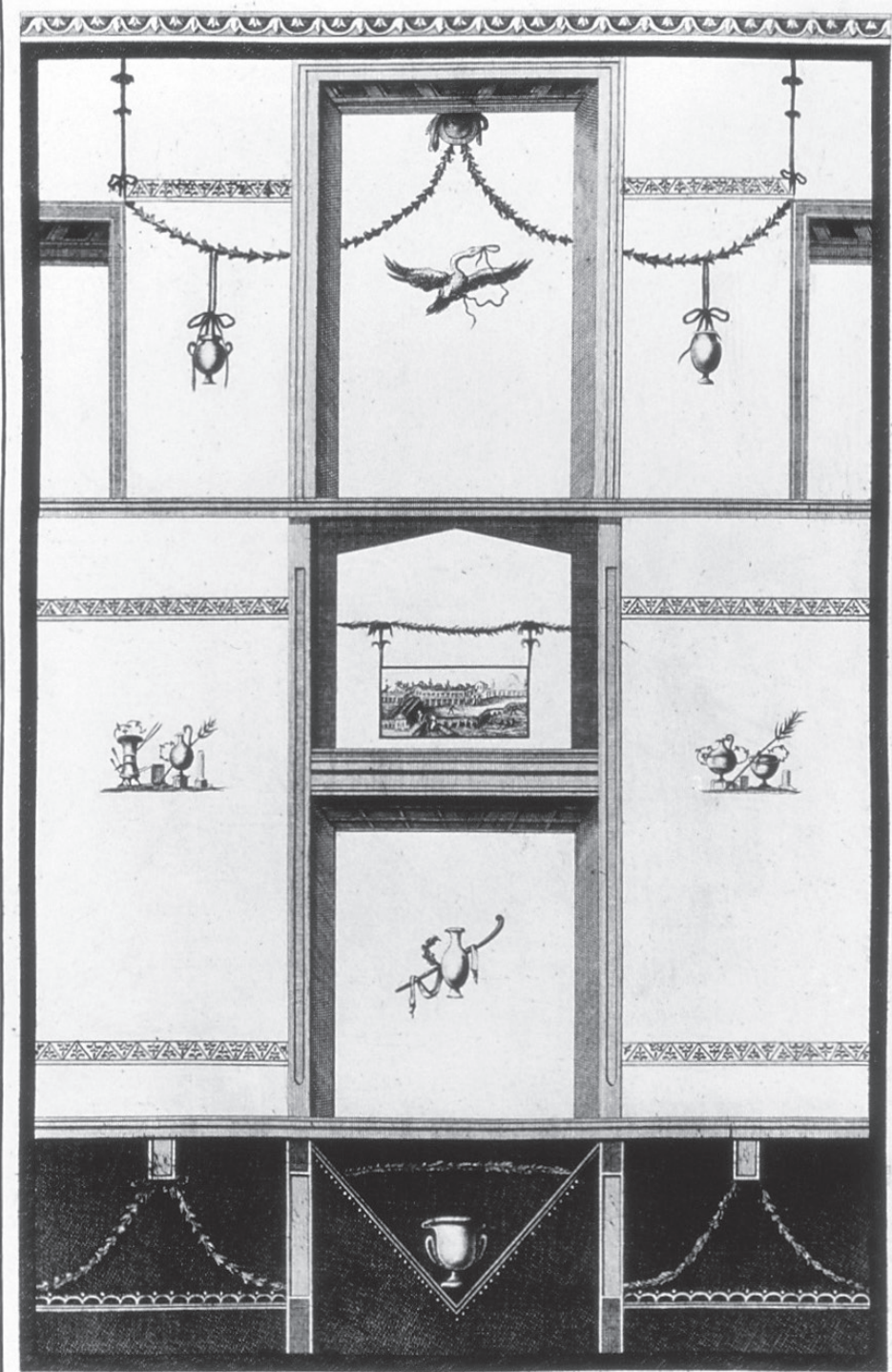

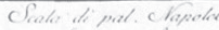

Fig. 2. Castellammare di Stabia, Villa San Marco, stanza 52, parete Sud Da: Gli ornati delle pareti ed i pavimenti delle stanze dell'antica Pompei, incisi in rame, vol. II., Napoli 1808, tav. 26. 
Che esistessero piante molto dettagliate, lo provano quelle disegnate da Pierre Bardet (1744), conservate nell'Archivio di Stato a Napoli, che mostrano la planimetria di una parte della città di Ercolano e la planimetria e il profilo della cd. basilica. ${ }^{6}$ (figg. 3-4) Pare però che già nel Settecento la documentazione fosse lacunosa. Francesco La Vega, quando fu chiamato a dirigere gli scavi dopo la morte di Carlo Weber nel 1764, si mise a cercare le piante disegnate dal suo predecessore, ovviamente senza trovarle. ${ }^{7}$ La Vega eseguì una planimetria di Ercolano molto sommaria, che prova la mancanza di quelle disegnate prima. ${ }^{8}$ (Fig.5)

In queste circostanze, con le difficoltà degli scavi sotterranei, non c'è da meravigliarsi se i rapporti di scavo spesso non permettono l'identificazione né del luogo di scavo né degli oggetti ritrovati. Come indicazione topografica, al massimo viene segnalato il "pozzo" attraverso il quale si scese alla città sotterranea, oppure il nome del proprietario del terreno - informazione, che ad Ercolano aiuta poco, perché si scavava quasi sempre "sotto la masseria del Bisogno" la quale copriva quasi tutto il terreno al di sopra della città antica. ${ }^{9}$

Sono appunto le pitture murali che aiutano ad individuare i luoghi di scavo menzionati nei documenti settecenteschi. Se si riesce ad individuare un dipinto descritto nei documenti di scavo e a ritrovarne in situ la collocazione originaria, i rapporti di scavo possono offrirci altre informazioni preziose, anche a proposito del rinvenimento di statue ed oggetti.

Esempio emblematico è la Basilica Noniana. ${ }^{10}$ Il 23 giugno 1739 fu staccata una piccola pittura, parte di un fregio a fondo nero con una ghirlanda in un vaso

6 Fonti documentarie per la storia degli scavi di Pompei Ercolano e Stabia. A cura degli archivisti napoletani. Napoli, 1979. A. Allroggen-Bedel, “ Dokumente des 18. Jahrhunderts zur Topographie von Herculaneum ", CErc 13, 1983, pp. 140-141 figg. 1-2. A. Allroggen-Bedel, “A proposito dei Balbi: note archivistiche alla topografia d'Ercolano ", in C. Gasparri, G. Greco, R. Pierobon Benoit (a cura di), Dall'immagine alla storia. Studi per ricordare Stefania Adamo Muscettola, Quaderni del Centro Studi Magna Grecia 10, Napoli 2010, figg. 4-6.8.

7 C.C.Parslow,Rediscovering Antiquity. KarlWeberandthe ExcavationofHerculaneum, Pompeii, and Stabiae, Cambridge 1995, pp. 271-277. Allroggen-Bedel, “ Dokumente des 18. Jahrhunderts ", pp. 359-361.

8 C. Rosini, Dissertationis isagogicae ad Herculanensium voluminum explanationem Pars prima, Napoli 1797, tav. 2.

9 M. Ruggiero, Storia degli scavi di Ercolano, tav. 2.

10 A. Allroggen-Bedel, "Das sogenannte Forum von Herculaneum und die borbonischen Grabungen von 1739 », CErc 4, 1974, pp. 97-109. A. Allroggen-Bedel, “ La Basilica Noniana », in M. P. Guidobaldi (a cura di), Ercolano. Tre secoli di scoperte, Napoli 2008, pp. 46-53. A. Allroggen-Bedel, “ A proposito dei Balbi », pp. 355-357. 


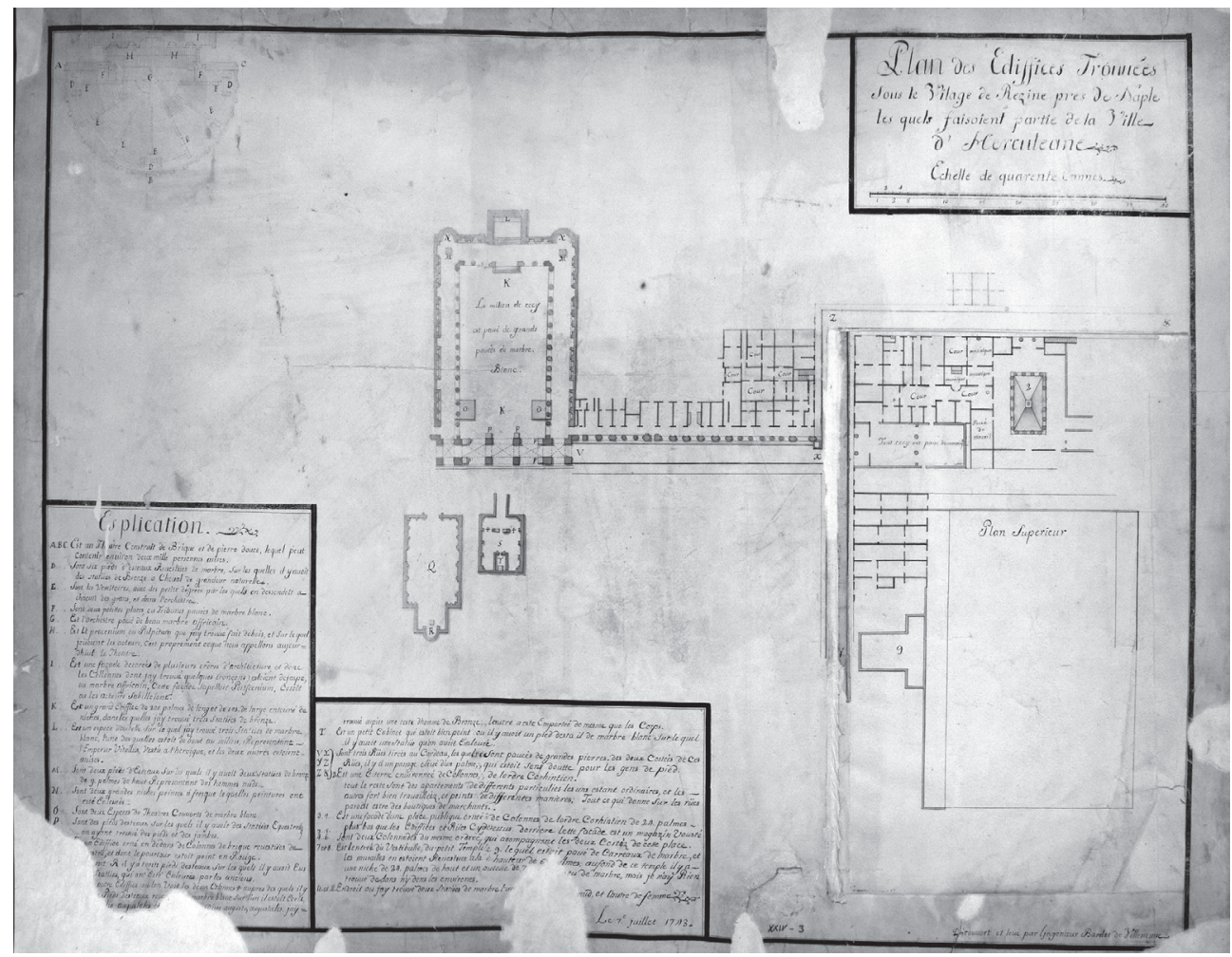

Fig. 3. Ercolano, planimetria di Pierre Bardet (1744)

Archivio di Stato Napoli, raccolta piante e disegni, cart. XXIV n. 3.

e due capretti. L'identificazione di questo dipinto staccato e portato al museo ha reso possibile il recupero (prima quasi del tutto virtuale e successivamente confermato dai saggi recenti) del complesso monumentale della Basilica Noniana con il suo allestimento scultoreo, aggiungendo alla ben nota cd. Basilica o Augusteum un altro complesso importante per la conoscenza dell'architettura e della società ercolanese.

La pittura in situ - abbastanza mal conservata, oggi non più visibile - prova che la pittura descritta nel rapporto di scavo alla data del 23 giugno 1739, fu staccata dalla parete di questo edificio. Sappiamo, quindi, che nel giugno 1739 gli scavatori lavoravano esattamente in questo punto. Si tratta della "nueva escavacion" menzionata nei documenti e ciò prova che le statue e le iscrizioni trovate nella "nueva escavacion" non provengono dalla cd. Basilica Augusteum. Siamo quindi in grado di definire meglio il programma scultoreo sia della cd. Basilica, sia della Basilica Noniana; le analisi e le interpretazioni possono partire dall'idea che vi erano due gruppi di statue, quello della famiglia dei Balbi nella 


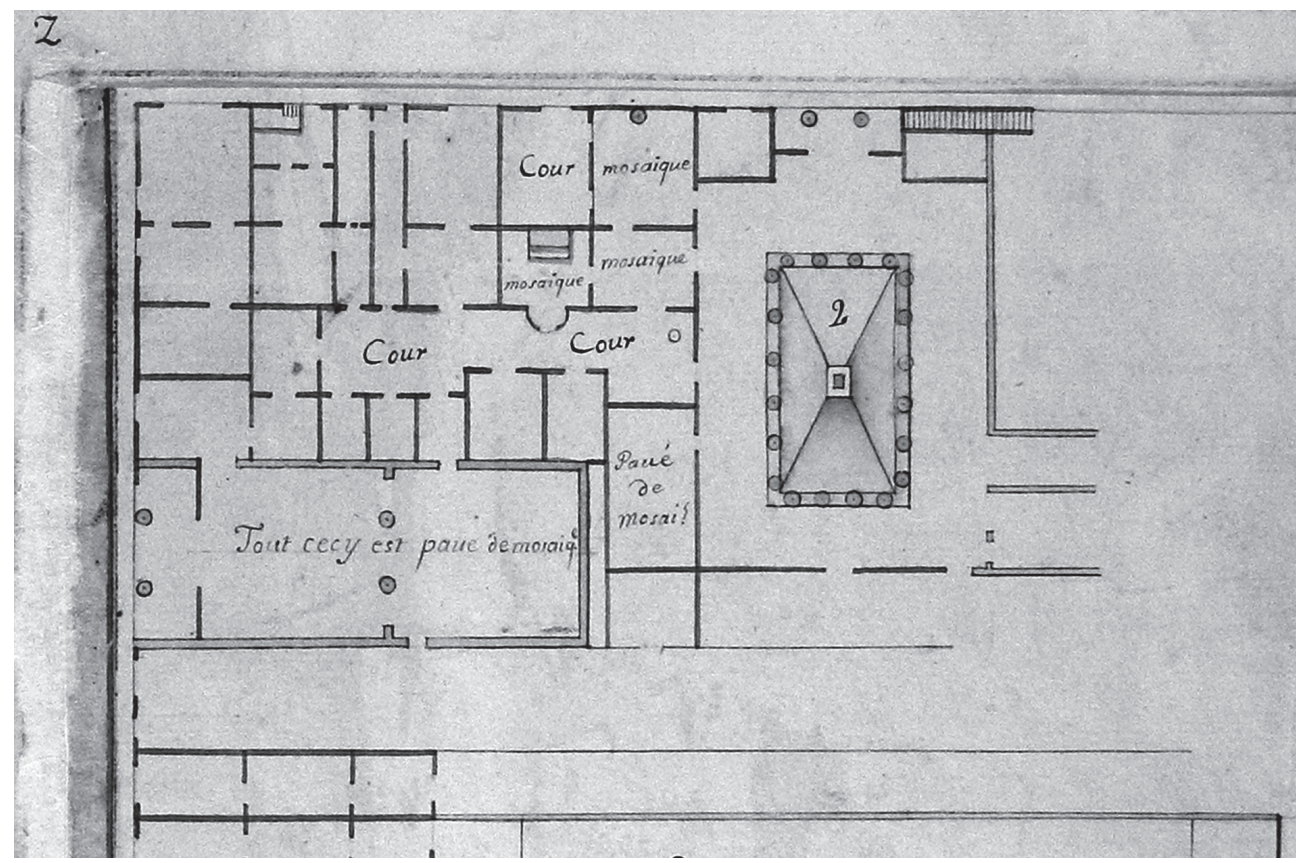

Fig. 4. Ercolano, planimetria di Pierre Bardet (1744): insula al di sopra della 'palestra'

Archivio di Stato Napoli, raccolta piante e disegni, cart. XXIV n. 3 (dettaglio)

Basilica Noniana, e quello della famiglia imperiale nella cd. Basilica Augusteum, come hanno dimostrato saggi recenti. ${ }^{11}$

I ritrovamenti documentati alla stessa data però non provengono sempre necessariamente dallo stesso posto. Spesso si scavò contemporaneamente in luoghi diversi, talvolta molto distanti fra di loro, come nel caso degli scavi eseguiti contemporaneamente nel teatro e nella "nueva escavacion". ${ }^{12}$

Il rapporto del 17 maggio 1746 menziona „tres lugares“, nei quali si scavarono: il luogo "del cavallo" e due case. ${ }^{13}$ L'espressione "del cavallo" si riferisce alla zona del foro, vicino al teatro, dove si trovavano tra l'altro le famose statue equestri di Marcus Nonius Balbus. ${ }^{14} \mathrm{La}$ "casa con pitture" è da identificare con un complesso di terzo stile nella Casa Ins. Or. II 2, dove dal 24 aprile al 9 maggio 1746 furono

1 D. Esposito, D. Camardo, “ La ‘Basilica Noniana' di Ercolano », MDAIR 119, 2013, 221258.

12 A. Allroggen-Bedel, « Das sogenannte Forum », p. 97-107.

13 A. Allroggen-Bedel, « A proposito dei Balbi », p. 368.

14 A. Allroggen-Bedel, «A proposito dei Balbi », p. 368-370. 


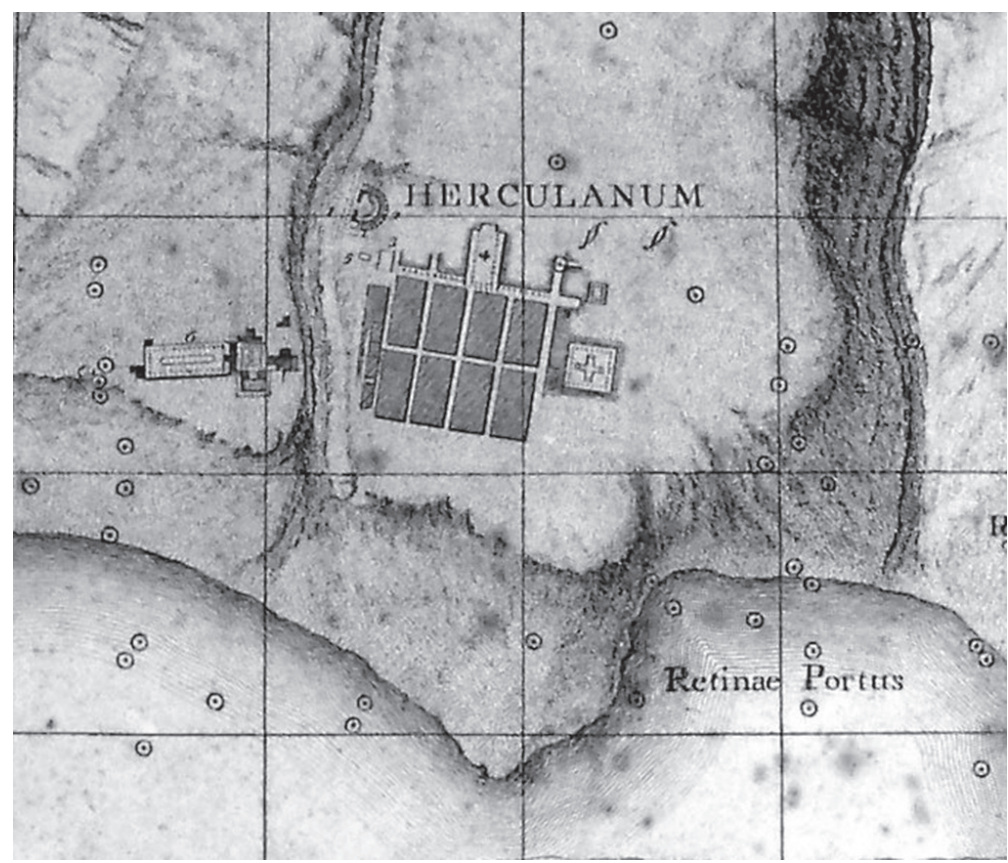

Fig. 5. Topografia dei Villaggi di Portici, Resina, e Torre del Greco, e di porzione de' loro territori levata sotto la direzione di Francesco La Vega e disegnata da Pietro La Vega (dettaglio). Napoli, Biblioteca Nazionale "Vittorio Emanuele III".

staccate complessivamente 22 pitture, portate al museo. ${ }^{15}$ L'altra casa non è stata riconosciuta; dato che i ritrovamenti non presentano alcuna caratteristica peculiare tale da consentirne l'identificazione. Ciò dimostra che non basta la data di scavo per stabilire la provenienza di una pittura o di un oggetto.

Nei rapporti di scavo spesso le pitture staccate sono descritte in modo molto approssimativo, con l'indicazione del soggetto, come ad esempio "puttino in aria", "donna velata", "uccello" ecc. Solo raramente sono indicati i colori della parete sulla quale erano dipinti questi soggetti. Il repertorio ripetitivo delle decorazioni murali rende ancora più difficile l'identificazione dei motivi descritti nelle relazioni di scavo. È quasi impossibile riconoscerli se non ci sono altre informazioni oppure segni distintivi. Infatti si possono individuare soprattutto i quadri figurati, e le scene mitologiche.

15 A. Allroggen-Bedel, “ Dokumente des 18. Jahrhunderts », p. 154 nota 81. E. M. Moormann, "Un fior di giardino ed altri frammenti di pittura ercolanesi ", CErC 16, 1986, pp. 123-133. 
Nel rapporto del 25 febbraio 1746 viene descritta una pittura con quattro persone e un cavallo, che corrisponde al quadro con Fedra ed Ippolito, conservato nel museo (9041). Di una seconda pittura, trovata lo stesso giorno, è riportata solo la notizia che si tratta di "due figure mal conservate", della stessa misura. Anche questo dipinto si trova nel museo (8601) sebbene abbastanza malridotto. Sono, infatti, appena identificabili due figure, interpretate nel catalogo del 1756 come Narciso ed Eco. Ė' però ipotizzabile che vi si debbano riconoscere Perseus e Andromeda.

La localizzazione dei due quadri e di un gruppo di pitture è stata possibile grazie alla identificazione di un piccolo frammento della decorazione parietale, rimasto in situ nella zona superiore del triclinio della Casa di Nettuno e Anfitrite (VI 6-7,7). ${ }^{16}$ Si tratta dell'angolo di un'edicola che permette di individuare il pendant in una pittura oggi al museo: dove si tratta di un'edicola con una figura giovanile, alla quale si aggiungono altri personaggi. Il risultato è un insieme di figure che corrispondono alla situazione in situ e che appartenevano alla zona superiore della decorazione. Grazie ad alcune caratteristiche peculiari possono essere individuate tra le pitture elencate da Alcubierre, nel periodo dal 22 febbraio al 21 marzo 1746, insieme ai due quadri mitologici e a due donne volanti. Le attribuzioni ottenute sulla base dei confronti tra le pitture nel museo e quelle in situ sono ulteriormente confermate dai documenti d'archivio e permettono di attribuire alla casa anche altri frammenti.

Il tablino nella Casa dell'Atrio Corinzio (V 30, 2) costituisce un caso simile: i resti in situ permettono l'attribuzione alla domus di una serie di pitture custodite nel museo, i documenti d'archivio ne danno conferma e permettono di aggiungere anche due quadri mitologici originariamente posti al centro delle pareti. ${ }^{17}$

16 A. Dardenay, A. Allroggen-Bedel, H. Eristov, M.-L. Maraval, N. Monteix, « Habitat et société à Herculanum », Chronique des activités archéologiques de l'École française de Rome [En ligne], « Les cités vésuviennes », mis en ligne le 15 avril 2015, consulté le 12 mai 2017. URL : http://cefr.revues.org/1339

17 A. Altroggen-Bedel, “ Lokalstile in der campanischen Wandmalerei », in Kölner Jahrbuch für Vor- und Frühgeschichte 24, 1991, pp. 35-41. A. Allroggen-Bedel, " Ercolano: dal sito al museo, dal museo agli archivi. Il puzzle ercolanese », in A. Coralini (a cura di), Vesuviana. Archeologie a confronto. Atti del Convegno Internazionale (Bologna, 14-16 gennaio 2008), Bologna 2009, pp. 167-179 figg. 4-5. A. Dardenay, H. Eristov, M.-L. Maraval, "Habitat et société à Herculanum ", Chronique des activités archéologiques de l'École française de Rome [En ligne], Les cités vésuviennes, mis en ligne le 12 juin 2014, consulté le 12 mai 2017. URL : http:// cefr.revues.org/ 1222 
Lo stesso vale per l'esedra nella Casa dell'Atrio a Mosaico (IV 1-2, 9): sulla base delle evidenze in situ e nel museo sono da individuare complessivamente 21 frammenti staccati, elencati nella relazione di scavo tra il 6 e il 13 agosto 1748, che conferma soprattutto la provenienza di un quadro mitologico con la rappresentazione di Paride sull'Ida (9508), staccato dal centro della parete Est. ${ }^{18}$ Pure nel caso di un oecus nella Casa dei Cervi (IV 21, 16), dal quale provengono otto nature morte di altissima qualità $(8615,8644,8647)$, la provenienza viene confermata dalla relazione di scavo. ${ }^{19}$

La presenza degli scavatori settecenteschi nella Casa d'Argo risulta dal confronto tra alcune pitture murali custodite nel Museo di Napoli ed i resti della decorazione murale rimasti in situ. ${ }^{20} \mathrm{Nel}$ museo si trova un frammento bellissimo a fondo bianco con la parte superiore di un'edicola e una tenda di colore blu, orlato da un tralcio d'uva (9863) ${ }^{21}$. Nell'ambiente 17 della Casa d'Argo $($ II 2,17$)$ si vedono ancora i resti di una decorazione che mostra lo stesso motivo permettendo l'attribuzione del frammento nel museo alla zona superiore. La tenda orlata con tralci d'uva corrisponde alla descrizione di una pittura, riportata da Alcubierre il 16 ed il 19 ottobre 1745: „Un leño enlazado de hojas de Parro, y uvas“22. I resti della decorazione in situ, la pittura nel museo e la notizia di Alcubierre confermano la presenza degli scavatori borbonici nella Casa d'Argo tra il 16 ed il 19 ottobre 1745.

Dalla stessa stanza provengono due pitture con pavoni su una specie di tripode (8759). Sul frammento più grande a sinistra sono visibili i resti di un

18 A. Allroggen-Bedel, « Lokalstile », p. 36 n. 21. Pannuti, “ Diario di scavo », pp. 261263. A. Allroggen-Bedel, "Zeitstil und Lokalstil: Überlegungen zur Methodik », in N. Zimmermann (Hrsg.), Wandmalerei zwischen Lokalstil und Zeitstil. Akten des XI. Internationalen Kolloquiums der AIPMA (Association pour la Peinture Murale Antique) 13.-17. September 2010 in Ephesos. Denkschriften der philosophischhistorischen Klasse, Bd: 468. Archäologische Forschungen, Bd: 23. Wien 2015, pp. 41-53.

19 A. Allroggen-Bedel, « Der Hausherr der „Casa dei Cervi“ in Herculaneum », CErc 5, 1975, pp. 102-103.

20 A. Dardenay, A. Allroggen-Bedel, H. Eristov, A. Grand-Clément, M.-L. Maraval, C. Marotta, N. Monteix, E. Rosso, “ Herculanum. Des archives aux restitutions architecturales et décoratives ", in Chronique des activités archéologiques de l'École française de Rome [En ligne], Les cités vésuviennes, mis en ligne le 09 juin 2016, consulté le 09 juin 2016. URL : http://cefr.revues.org/1588

21 R. Herbig, Nugae Pompeianorum, p. 13 tav. 23-26. D. Esposito, La pittura di Ercolano, Studi della Soprintendenza Archeologica di Pompei 33, Napoli 2014 (frontispizio).

22 M. Pagano, R. Prisciandaro, Studio sulle provenienze, p. 60 (con traduzione sbagliata: „ramo intrecciato con tralci e uva“). 
tralcio d'uva, che corrisponde perfettamente a quello che incornicia la tenda blu dell'altro frammento. Come risulta dalle pitture rimaste in situ, i due frammenti si trovavano ai lati dell'edicola centrale.

In un altro ambiente della stessa casa furono staccati due frammenti con maschere tragiche (9821), i cui compagni rimasti in situ nella stanza 20 permettono la localizzazione dei due frammenti staccati nella zona superiore della parete $\operatorname{sud}^{23}$.

La data del loro ritrovamento non è nota. Secondo Mario Pagano e Raffaele Prisciandaro sono da riconoscere tra le 15 pitture elencate in un allegato ad una lettera del 13 settembre $1745^{24}$. Qui figurano infatti due maschere, collocate insieme in una delle 12 "casse". Si tratta di "quattro mascheroni di lunghezza pal. 2 oncie 7, e d'altezza oncie 8", le cui misure però non corrispondono ai due frammenti provenienti dalla Casa d'Argo ${ }^{25}$. L'elenco citato non riporta nessuna data, ma fornisce solo un terminus ante quem per il ritrovamento delle pitture, grazie alla lettera d'accompagnamento. ${ }^{26}$ Nei rapporti di Alcubierre di quel periodo non si trovano rappresentazioni di maschere. Nel settembre 1745 si lavorava invece a staccare una grande quantità di pitture appartenenti ad un bel complesso di Terzo stile, che sappiamo si trovava non tanto lontano dal teatro ${ }^{27}$.

Con lo stesso metodo si possono individuare gruppi di pitture staccate appartenenti agli stessi complessi decorativi, forse ancora seppelliti oppure distrutti. La nota architettura molto delicata ("goldschmiedehaft") dipinta su un bel fondo rosso (9878), mostra delle caratteristiche che permettono di aggiungere una serie di altre pitture.$^{28} \mathrm{Il}$ contesto viene evidenziato in parte dai motivi, come ad es. le figure in piedi, situate su finissimi piedistalli. Un altro gruppo di pitture consiste in una serie di fregi a fondo nero, rappresentanti scene mitologiche oppure dionisiache. I colori, lo stile e soprattutto alcuni dettagli ornamentali, come ad es. la striscia bianca con linee blu, permettono di riconoscerli come provenienti da un unico complesso decorativo.

23 A. Allroggen-Bedel, Maskendarstellungen in der römisch-kampanischen Wandmalerei, München 1974, pp. 123-124 figg. 6-9.

24 M. Pagano, R. Prisciandaro, Studio sulle provenienze, p. 55.

25 M. Pagano, R. Prisciandaro, Studio sulle provenienze, p. 55 : lunghezza $68,2 \mathrm{~cm}$, altezza 17,6 cm. MANN 9821: lunghezza $63 \mathrm{~cm}$, altezza $12 \mathrm{~cm}$.

26 M. Pagano-R. Prisciandaro individuano il 13 settembre 1745 come data di ritrovamento. M. Pagano, R. Prisciandaro, Studio sulle provenienze, p. 55.

27 A. Allroggen-Bedel, " Ercolano: dal sito al museo », pp. 173-177 figg. 6-9.

28 A. Allroggen-Bedel, “ Ercolano: dal sito al museo », pp. 173-177 figg. 6-9. 
Dal rapporto di scavo del 1745 che fornisce una descrizione abbastanza dettagliata delle pitture staccate, possiamo dedurre che i due gruppi - sia quello a fondo rosso con l'architettura e le figure danzanti, sia quello con i fregi figurati a fondo nero - furono trovati contemporaneamente e nello stesso luogo. ${ }^{29}$

Pure l'attribuzione delle famose scene isiache (8919 e 8924) a questo complesso è dovuta al rapporto di scavo che ne fornisce una descrizione esplicita. ${ }^{30} \mathrm{Il}$ fatto che facessero parte di questo complesso decorativo è importante per l'inquadramento stilistico, sia delle architetture che dei fregi, dei quadretti e degli altri motivi.

Nonostante il numero cospicuo di pitture che sono da attribuire a questo complesso, grazie ai motivi e alla relazione di scavo, i dati noti non sono sufficienti comunque per una ricostruzione anche se ci danno un'idea di quel che deve essere stato questo complesso. Sebbene pare ovvio che le pitture trovate dal 13 settembre al 4 ottobre 1745 appartengano allo stesso complesso decorativo, non provengono necessariamente dalla stessa stanza. Come si può constatare nelle case meglio conservate, un "complesso decorativo" poteva comprendere più di un ambiente. Esempio tipico sono le pitture di terzo stile nella villa in Campo Varano: come hanno mostrato gli ultimi scavi, non è detto che pitture dello stesso stile con motivi molto simili provengano sempre dallo stesso ambiente.

Purtroppo non sappiamo dove sia stato trovato questo bellissimo complesso pittorico, né da quale edificio fu asportato. Dal rapporto di scavo risulta però che nei giorni dello scavo una pioggia molto forte costrinse gli operai a mettersi al sicuro, uscendo da un cunicolo vicino al teatro. ${ }^{31}$ Quindi è da supporre che gli scavi abbiano avuto luogo non tanto lontano dal teatro, in una zona non ancora messa in luce.

In un altro caso lo studio della provenienza, basata sull'evidenza di una ventina di frammenti custoditi nel museo e sulla relazione di scavo, ci fornisce dei dati su un complesso di altissima qualità artistica, che prova l'esistenza di una villa situata fuori dalla città. ${ }^{32}$

29 U. Pannuti, « Diario di scavo », pp. 218-221.

30 U. Pannuti, « Diario di scavo »,p. 219 (8919: 18 settembre 1745). U. Pannuti, « Diario di scavo ", p. 218 (8924: 13 settembre 1745).

31 U. Pannuti, “ Diario di scavo », p. 219 (22 settembre 1745).

32 A. Allroggen-Bedel, "Die Wanddekorationen der Villen am Golf von Neapel », in La regione sotterrata del Vesuvio. Studi e prospettive (Atti del convegno internazionale 11-15 novembre 1979), Napoli 1982, p. 520. 
Si tratta di una serie di bellissime pitture su fondo nero, tra le quali la famosa rappresentazione di una testa femminile, vista di profilo, ritenuta un ritratto (9077)..33 Gli altri dipinti mostrano vari uccelli, frutta, un tralcio. Sono tutti da individuare nel museo di Napoli, ma purtroppo i frammenti non bastano per farsi un'idea della decorazione.

Il busto femminile dovrebbe appartenere alla zona superiore in quanto, sebbene il disegno geometrico potrebbe essere attribuito anche allo zoccolo, il formato e la qualità eccezionale lasciano supporre che abbia occupato un posto più importante nel contesto decorativo. La donna, riccamente ornata con orecchini, potrebbe essere una divinità, ma le sue sembianze non escludono che possa essere riconosciuto come ritratto di una raffinata domina. Un busto simile ma di un formato molto più piccolo è raffigurato nelle decorazioni della villa di Boscotrecase.

Dalle relazioni di scavo risulta che da aprile a maggio 1752 si scavava vicino alla chiesa di Santa Maria di Pugliano, a monte di Resina, in un luogo chiamato "el Fosso de Callolla", "junto al piè del Vesuvio, è imediato á Portici". ${ }^{34}$ Le pitture non vennero staccate tutte insieme nello stesso giorno, ma il distacco ebbe luogo il 23 e il 30 aprile, e il 21 e 28 maggio 1752. ${ }^{35}$ Non è nemmeno possibile stabilire se appartengano alla decorazione di un unico ambiente oppure se si tratti di pitture provenienti da vari ambienti.

L'individuazione delle pitture provenienti da questo scavo settecentesco restituisce non solo i resti di un bellissimo complesso pittorico, sebbene abbastanza lacunoso, ma fornisce anche un'importante informazione sulla topografia ercolanese. L'alta qualità delle pitture lascia immaginare una dimora signorile della quale purtroppo fino ad oggi non abbiamo altri dati.

Come dimostrano gli esempi presi in esame, i documenti d'archivio sono strumenti importanti per la ricostituzione dei complessi decorativi. Ł̀ fondamentale però l'evidenza dei rinvenimenti, in situ e nei musei che costituisce la base per la lettura e l'interpretazione dei documenti settecenteschi. Senza spiegare poi il ragionamento che ha portato all'attribuzione di una pittura

33 R. Herbig, Nugae Pompeianorum. Unbekannte Wandmalereien des dritten Pompejanischen Stils, Tübingen 1962, pp. 19-20..

34 U. Pannuti, “ Diario di scavo », pp. 304-306 (23 aprile-28 maggio 1752). A. AllroggenBedel, “Die Wanddekorationen der Villen », p. 520. L.A.Scatozza Нöricht, “Ville nel territorio ercolanese », CrErc 15, 1985, p. 162.

35 Primo gruppo, 23-30 aprile 1752 (U. Pannuti, “ Diario di scavo », p. 304): MANN 9077, 8623, 8867, 8736 sotto, 8742, 8750, 8751, 9813; secondo gruppo, 21-28 maggio 1752 (Pannuti, “ Diario di scavo », pp. 305-306): 8761, 9964, 8757, 9779, 8745, 8731, 8736 (sopra), 9811, Inv. Avellino 71. 
oppure di un oggetto ad una casa o ad un ambiente, c'è il rischio di presentare il risultato di un ragionamento o di una catena di ragionamenti, come un fatto, non come un'ipotesi. ${ }^{36}$ Dobbiamo renderci conto della differenza tra la provenienza di un oggetto recuperato in situ, fotografato, messo in deposito con un numero d'inventario - e la provenienza di un oggetto descritto in un documento settecentesco, individuato nel museo e forse, grazie ad una ricerca fatta sul sito, attribuito ad una casa, un ambiente, una parete. In questi casi è decisivo spiegare quanto è affidabile la ricostruzione oppure l'ipotesi.

Usati ed interpretati in questo modo, i documenti di scavo sono davvero strumenti preziosi per il recupero dei complessi decorativi ercolanesi.

\section{Agnes Allroggen-Bedel}

chercheuse indépendante associée au programme ANR VESUVIA

riess-ab@online.de

36 Per l'inserimento nel database il problema è che è possibile solo riportare un'informazione sintetica. Sarebbe necessario il riferimento a pubblicazioni più ampie con tutti i ragionamenti che portavano alla localizzazione per poter capire l'argomentazione e soprattutto per poter controllare il risultato. Gli articoli pubblicati durante gli ultimi anni, "Les chroniques" del progetto VESUVIA, ne sono un esempio. 